\title{
Influence of hydrogen absorption on low-energy electronic collective excitations in palladium
}

\author{
V. M. Silkin, ${ }^{1}$ I. P. Chernov, ${ }^{2}$ P. M. Echenique,,${ }^{1,3}$ Yu. M. Koroteev, ${ }^{4}$ and E. V. Chulkov ${ }^{1,3}$ \\ ${ }^{1}$ Donostia International Physics Center (DIPC), Paseo de Manuel Lardizabal 4, 20018 San Sebastián, Basque Country, Spain \\ ${ }^{2}$ Tomsk Polytechnical University, Prospekt Lenina 30, 634050 Tomsk, Russia \\ ${ }^{3}$ Departamento de Física de Materiales and Centro Mixto CSIC-UPV/EHU, Facultad de Ciencias Químicas, Universidad del País Vasco, \\ Apartado 1072, 20080 San Sebastián, Basque Country, Spain \\ ${ }^{4}$ Institute of Strength Physics and Materials Science, Russian Academy of Sciences, Prospekt Academicheskii 2/1, 634021 Tomsk, Russia
}

(Received 26 June 2007; revised manuscript received 22 October 2007; published 6 December 2007)

\begin{abstract}
A theoretical study of electronic excitation spectra in $\mathrm{Pd}$ and $\mathrm{PdH}$ is reported. The calculations were performed with full inclusion of the electron band structure obtained within self-consistent pseudopotential approach. We demonstrate that the complicated Pd electronic structure at the Fermi level is reflected in a numerous peak structure of the excitation spectra. The evolution of the energy-loss spectrum with momentum and its anisotropy are analyzed. Strong modification of the excitation spectra upon hydrogen absorption is found. We also study the role of intra- and interband transitions in the formation of dominating plasmon peak both in pure $\mathrm{Pd}$ and $\mathrm{PdH}$. In $\mathrm{Pd}$, this peak is mainly determined by intraband transitions. The downward shift of this peak from $\sim 7.2 \mathrm{eV}$ in pure $\mathrm{Pd}$ to $\sim 4.2 \mathrm{eV}$ in $\mathrm{PdH}$ is mainly explained by interband transitions from occupied $\mathrm{Pd} d$ bands to unoccupied hydrogen-modified $s p$ states in the $7-13 \mathrm{eV}$ energy range.
\end{abstract}

DOI: 10.1103/PhysRevB.76.245105

PACS number(s): 71.20.Be, 71.15.Mb, 71.45.Gm

\section{INTRODUCTION}

The physical properties of metal-hydrogen systems have extensively been studied over many years. ${ }^{1-3}$ These systems are interesting both from practical and theoretical points of view. They are important in energy-storage systems, in sensor devices, in catalysis, as well as in other fields of modern technology. The presence of hydrogen in metals significantly influences their transport, mechanical, electrical, magnetic, and other properties. The remarkable example of this is the palladium-hydrogen systems which attracted attention for many decades due to peculiar properties. ${ }^{4}$ The change of physical properties of metals upon hydrogen absorption is closely related to strong modification of electronic structure of the materials. ${ }^{5-9}$ In the case of palladium, the hydrogen absorption leads to three major effects in $\mathrm{PdH}_{x}$ : (i) appearance of hydrogen induced electron states below valence $d$ band, (ii) reduction of the width of valence $d$ band, and (iii) sinking of this $d$ band relative to the Fermi level with increase of $\mathrm{H}$ concentration that results in drastic reduction of density of states at the Fermi level at $x \geqslant 0.6$. The modification of electronic structure is reflected in excitation spectra which contain information on single-particle and collective excitations in the system. Extensive work on collective excitations in palladium has been done with the use of electronenergy-loss spectroscopy ${ }^{5,10-13}$ (EELS) (see also data on optical spectra of palladium in Refs. 7 and 14).

The interpretation of experimental electron-energy-loss spectra is a rather difficult task, as the data can strongly be influenced by the surface excitations. Therefore, there is a noticeable uncertainty in the interpretation of experimentally observed peaks. ${ }^{13}$ Nevertheless, the majority of experimental conclusions $^{11-13}$ and first-principles calculations ${ }^{15-17}$ agree: The dominant peak found in the energy range from 7 to $8 \mathrm{eV}$ is the bulk plasmon peak and the others are due to interband transitions (here, we refer to the valence electron energy range only, i.e., below the core electron excitations).

Momentum dependence of electron-energy-loss spectra in Pd has been studied by Netzer and El Gomani in EELS measurements. ${ }^{18}$ They observed four features in the energy interval up to $10 \mathrm{eV}$. The dominant peak at $\sim 6.6 \mathrm{eV}$ was accounted for by the overlap of the surface and bulk plasmons. This peak shows weak dispersion to higher energy with increasing momentum. A shoulder at $\sim 4 \mathrm{eV}$ (interpreted as associated with interband transition) disappears already at small momenta. As to other broad features, no conclusion about their dispersion has been done.

In EELS measurements of collective excitations in $\mathrm{PdH}_{0.8}$, a shift of the dominant plasmon peak from around $7 \mathrm{eV}$ in $\mathrm{Pd}$ to around $5 \mathrm{eV}$ in $\mathrm{PdH}_{0.8}$ has been found. ${ }^{5}$ The significant change contradicts a simple picture based on a free-electron gas model where the only parameter determining the system property is an average electron density $n_{0}$. This density is slightly higher in $\mathrm{PdH}_{0.8}$ than in clean $\mathrm{Pd}$. Following the free-electron gas model, this should lead to slight increase of the plasmon energy instead of almost 30\% reduction observed experimentally. The electronic structure of pure Pd around the Fermi level is dominated by $4 d$ states which form a strong peak in density of states at the Fermi level. The modification of electronic structure upon hydrogen absorption has been studied in a number of first-principles calculations; ${ }^{8,9,19-22}$ however, we are not aware of the corresponding $a b$ initio calculations of the excitation spectra in palladium hydrides, although it was done for some other metal hydrides. ${ }^{23-25}$ To our knowledge, theoretical investigations of anisotropic properties of the excitation spectra have not been reported either.

The purpose of the present paper is the ab initio study of the excitation spectra in pure Pd and $\mathrm{PdH}$. The energy-loss spectra for both systems are investigated for three symmetry directions in the low-energy domain. The role played by crystal local-field effects is analyzed as well. The paper is organized as follows. In Sec. II, the calculation procedure is described. In Sec. III, we present the main results which include the loss spectra for both pure Pd and PdH systems. Finally, the conclusions are drawn in Sec. IV. 


\section{CALCULATION DETAILS}

Within the first Born approximation, the inelastic scattering cross section of $\mathrm{x}$ rays and electrons for momentum transfer $\mathbf{q}$ and energy $\hbar \omega$ transferred to the system is proportional to the dynamical structure factor $S(\mathbf{q}, \omega)$ of manyelectron systems. $S(\mathbf{q}, \omega)$ is connected by the fluctuationdissipation theorem ${ }^{26}$ to the dielectric function $\epsilon\left(\mathbf{r}, \mathbf{r}^{\prime}, \omega\right)$ which, in general, is a nonlocal energy-dependent function. For a periodic solid, $S(\mathbf{q}, \omega)$ is

$$
S(\mathbf{q}, \omega)=-\frac{\hbar \Omega q^{2}}{2 \pi} \operatorname{Im}\left[\epsilon_{\mathbf{G}=0, \mathbf{G}^{\prime}=0}^{-1}(\mathbf{q}, \omega)\right],
$$

where $\Omega$ represents the normalization volume, G's are the reciprocal lattice vectors, and $\operatorname{Im}\left[\epsilon_{\mathbf{G}=0, \mathbf{G}^{\prime}=0}^{-1}(\mathbf{q}, \omega)\right]$ is the electron energy-loss function related to the Fourier coefficients of the density-response function $\chi_{\mathbf{G}, \mathbf{G}^{\prime}}(\mathbf{q}, \omega)$ through

$$
\epsilon_{\mathbf{G}, \mathbf{G}^{\prime}}^{-1}(\mathbf{q}, \omega)=\delta_{\mathbf{G}, \mathbf{G}^{\prime}}+v_{\mathbf{G}}(\mathbf{q}) \chi_{\mathbf{G}, \mathbf{G}^{\prime}}(\mathbf{q}, \omega) .
$$

Here, $v_{\mathbf{G}}(\mathbf{q})=4 \pi /|\mathbf{q}+\mathbf{G}|^{2}$ is the Fourier transform of the Coulomb potential. Within linear-response theory, $\chi$ relates the electron density $n^{\text {ind }}(\mathbf{r}, \omega)$ induced in the system to an external perturbation potential $V^{\text {ext }}(\mathbf{r}, \omega)$ through the equation

$$
n^{\text {ind }}(\mathbf{r}, \omega)=\int d \mathbf{r}^{\prime} \chi\left(\mathbf{r}, \mathbf{r}^{\prime}, \omega\right) V^{\text {ext }}\left(\mathbf{r}^{\prime}, \omega\right)
$$

In the framework of time-dependent density-functional theory, ${ }^{27,28} \chi\left(\mathbf{r}, \mathbf{r}^{\prime}, \omega\right)$ obeys the integral equation

$$
\begin{aligned}
\chi\left(\mathbf{r}, \mathbf{r}^{\prime}, \omega\right)= & \chi^{0}\left(\mathbf{r}, \mathbf{r}^{\prime}, \omega\right)+\int d \mathbf{r}_{1} \int d \mathbf{r}_{2} \chi^{0}\left(\mathbf{r}, \mathbf{r}_{1}, \omega\right) \\
& \times\left[v\left(\mathbf{r}_{1}-\mathbf{r}_{2}\right)+K^{\mathrm{xc}}\left(\mathbf{r}_{1}, \mathbf{r}_{2}, \omega\right)\right] \chi\left(\mathbf{r}_{2}, \mathbf{r}^{\prime}, \omega\right),
\end{aligned}
$$

where $\chi^{0}\left(\mathbf{r}, \mathbf{r}^{\prime}, \omega\right)$ is the density-response function for a noninteracting electron system, $v\left(\mathbf{r}-\mathbf{r}^{\prime}\right)$ is the bare Coulomb potential, and $K^{\mathrm{xc}}\left(\mathbf{r}, \mathbf{r}^{\prime}, \omega\right)$ accounts for dynamical exchangecorrelation effects. For a periodic crystal, all these quantities can be expanded in Fourier series and the integral equation (4) transforms into a matrix equation

$$
\begin{aligned}
\chi_{\mathbf{G}, \mathbf{G}^{\prime}}(\mathbf{q}, \omega)= & \chi_{\mathbf{G}_{\mathbf{G}^{\prime}}}^{0}(\mathbf{q}, \omega)+\sum_{\mathbf{G}_{1}} \sum_{\mathbf{G}_{2}} \chi_{\mathbf{G}_{\mathbf{G}}, \mathbf{G}_{1}}^{0}(\mathbf{q}, \omega) \\
& \times\left[v_{\mathbf{G}_{1}}(\mathbf{q}) \delta_{\mathbf{G}_{1}, \mathbf{G}_{2}}+K_{\mathbf{G}_{1}, \mathbf{G}_{2}}^{\mathrm{xc}}(\mathbf{q}, \omega)\right] \chi_{\mathbf{G}_{2}, \mathbf{G}^{\prime}}(\mathbf{q}, \omega)
\end{aligned}
$$

The Fourier coefficients $\chi_{\mathbf{G}, \mathbf{G}^{\prime}}^{0}(\mathbf{q}, \omega)$ have the form

$$
\chi_{\mathbf{G}, \mathbf{G}^{\prime}}^{0}(\mathbf{q}, \omega)=\frac{2}{\Omega} \sum_{\mathbf{k}}^{B Z} \sum_{n, n^{\prime}} \frac{\left(f_{n \mathbf{k}}-f_{n^{\prime} \mathbf{k}+\mathbf{q}}\right)\left\langle\psi_{n \mathbf{k}}\left|e^{-i(\mathbf{q}+\mathbf{G}) \cdot \mathbf{r}}\right| \psi_{n^{\prime} \mathbf{k}+\mathbf{q}}\right\rangle\left\langle\psi_{n^{\prime} \mathbf{k}+\mathbf{q}}\left|e^{i\left(\mathbf{q}+\mathbf{G}^{\prime}\right) \cdot \mathbf{r}}\right| \psi_{n \mathbf{k}}\right\rangle}{\varepsilon_{n \mathbf{k}}-\varepsilon_{n^{\prime} \mathbf{k}+\mathbf{q}}+\omega+i \eta},
$$

where the factor of 2 accounts for spin, the sums over $n$ and $n^{\prime}$ run over the band structure for wave vectors $\mathbf{k}$ in the first Brillouin zone (BZ), $f_{n \mathbf{k}}$ is the Fermi distribution function, $\varepsilon_{n \mathbf{k}}$ and $\psi_{n \mathbf{k}}(\mathbf{r})$ are Bloch eigenvalues and eigenfunctions of the Kohn-Sham Hamiltonian, and $\eta$ is positive infinitesimal. The numerical evaluation of $\chi_{\mathbf{G}, \mathbf{G}^{\prime}}^{0}(\mathbf{q}, \omega)$ matrix is the most time-demanding part of the calculations, and one should put some finite value for $\eta$ which introduces an additional broadening in calculated spectra. In practice, instead of direct use of Eq. (6), the matrix $\chi_{\mathbf{G}_{\mathbf{G}, \mathbf{G}^{\prime}}}^{0}(\mathbf{q}, \omega)$ can be evaluated by performing calculations at imaginary ${ }^{29}$ or complex ${ }^{30}$ frequencies $\omega$ with subsequent analytical continuation to the real axis. Here, we use an alternative route calculating at the first step the spectral function matrix $S_{\mathbf{G}, \mathbf{G}^{\prime}}^{0}(\mathbf{q}, \omega)$ as $^{31,32}$

$$
\begin{aligned}
S_{\mathbf{G}, \mathbf{G}^{\prime}}^{0}(\mathbf{q}, \omega)= & \frac{2}{\Omega} \sum_{\mathbf{k}}^{B Z} \sum_{n}^{\text {occ unocc }} \sum_{n^{\prime}}\left\langle\psi_{n \mathbf{k}}\left|e^{-i(\mathbf{q}+\mathbf{G}) \cdot \mathbf{r}}\right| \psi_{n^{\prime} \mathbf{k}+\mathbf{q}}\right\rangle \\
& \times\left\langle\psi_{n^{\prime} \mathbf{k}+\mathbf{q}}\left|e^{i\left(\mathbf{q}+\mathbf{G}^{\prime}\right) \cdot \mathbf{r}}\right| \psi_{n \mathbf{k}}\right\rangle \delta\left(\varepsilon_{n \mathbf{k}}-\varepsilon_{n^{\prime} \mathbf{k}+\mathbf{q}}+\omega\right),
\end{aligned}
$$

with subsequent evaluation of the imaginary part of $\chi_{\mathbf{G}, \mathbf{G}^{\prime}}^{0}(\mathbf{q}, \omega)$ via the relation

$$
S_{\mathbf{G}, \mathbf{G}^{\prime}}^{0}(\mathbf{q}, \omega)=-\frac{1}{\pi} \operatorname{sgn}(\omega) \operatorname{Im}\left[\chi_{\mathbf{G}_{\mathbf{G}} \mathbf{G}^{\prime}}^{0}(\mathbf{q}, \omega)\right]
$$

Here, $\operatorname{sgn}(\omega)=1(-1)$ for $\omega>0(\omega<0)$. The real part of the $\chi_{\mathbf{G}_{\mathbf{G}, \mathbf{G}^{\prime}}}^{0}(\mathbf{q}, \omega)$ matrix elements is obtained from the corresponding imaginary part by the Hilbert transform.

The present calculations have been performed for fcc $\mathrm{Pd}$ and the stoichiometric palladium hydride $\mathrm{PdH}$ in rocksalt structure where the palladium atoms form a fcc lattice and hydrogen atoms occupy all the octahedral sites. For Pd, we use the experimental lattice parameters $a_{\mathrm{Pd}}=7.3512$ a.u. Lattice parameter for $\mathrm{PdH}, a_{\mathrm{PdH}}$, has been taken as 7.7921 a.u., similar to Ref. 8 and close to the experimental value. ${ }^{33}$ This corresponds to the lattice expansion of $6 \%$ for $\mathrm{PdH}$ with respect to the pure $\mathrm{Pd}$. The one-particle energies $\varepsilon_{n \mathbf{k}}$ and wave functions $\psi_{n \mathbf{k}}(\mathbf{r})$ were obtained as a self-consistent solution of the Kohn-Sham equations with the use of exchangecorrelation potential in the form of Ref. 34. The electron-ion interaction was described by a nonlocal norm-conserving ionic pseudopotential for Pd (Ref. 35) and the hydrogen local ionic pseudopotential taken from Ref. 8. Wave functions $\psi_{n \mathbf{k}}(\mathbf{r})$ were expanded in plane waves up to a kinetic-energy cutoff of 70 Ry. Up to 40 reciprocal vectors $\mathbf{G}$ were included 


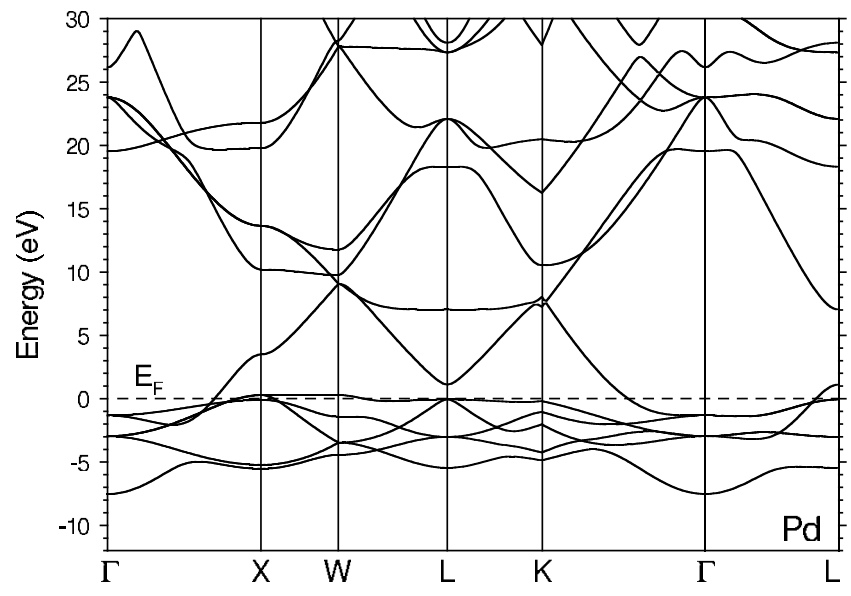

FIG. 1. The calculated band structure for pure Pd along some high symmetry directions of the Brillouin zone. The energies are according to the Fermi level shown by dashed horizontal line.

in the Fourier expansions of $\chi^{0}, \chi$, and $\epsilon$ matrices. Hence, the crystal local-field effects were incorporated in the evaluation of $\operatorname{Im}\left[\epsilon_{\mathbf{G}=0, \mathbf{G}^{\prime}=0}^{-1}(\mathbf{q}, \omega)\right]$ through the inclusion of nondiagonal matrix elements of $\chi_{\mathbf{G}_{\mathbf{G}, \mathbf{G}^{\prime}}^{0}}^{0}(\mathbf{q}, \omega)$ and matrix equation (5). In Eq. (7), the sum over $\mathbf{k}$ includes a $96 \times 96 \times 96$ sampling that corresponds to 442368 points in the BZ. The sums over $n$ and $n^{\prime}$ included all energy bands up to energy of $50 \mathrm{eV}$ above the Fermi level. The $S_{\mathbf{G}, \mathbf{G}^{\prime}}^{0}(\mathbf{q}, \omega)$ matrices were calculated at the discrete mesh of energies ranging from 0 to $50 \mathrm{eV}$ with a step of $0.05 \mathrm{eV}$. In the numerical calculations, the $\delta$ function in Eq. (7) was replaced by a Gaussian with a broadening of $0.1 \mathrm{eV}$. We solve Eq. (5) using a random-phase approximation, i.e., taking $K^{\mathrm{xc}}=0$. It was demonstrated in Ref. 36 that for transition metals, this is a rather good approximation for low-energy electronic excitations, an energy domain of interest here.

\section{CALCULATION RESULTS AND DISCUSSION}

\section{A. Pd}

As the basic ingredient of the dielectric matrix evaluation is the band structure, we plot it for Pd in Fig. 1. It agrees well with previous calculations, ${ }^{8,9,37,38}$ although some small quantitative differences in energy positions owing to the use of different calculation methods can be noted.

In Fig. 2, we present the calculated imaginary part of the inverse dielectric matrix, $\operatorname{Im}\left[\epsilon_{\mathbf{G}=0, \mathbf{G}^{\prime}=0}^{-1}(\mathbf{q}, \omega)\right]$, for pure $\mathrm{Pd}$ as a function of $\mathbf{q}$ and $\omega$ along the (100), (110), and (111) symmetry directions and for energies up to $15 \mathrm{eV}$. In order to enhance the fine structures in the low $\omega$ region, we scale $\operatorname{Im} \epsilon^{-1}$ by $\omega^{-1}$. Whereas this kind of presentation distorts to some extent the shape of the features, nevertheless, the positions of peak structures along the $\omega$ axis are not affected and it gives a general idea about the $\operatorname{Im} \epsilon^{-1}$ behavior in the $(\mathbf{q}, \omega)$-phase space. Figure 3 summarizes the peak positions in the calculated loss spectra of Pd along the three symmetry directions. From the figures, one can appreciate that the excitation spectra in Pd along all the symmetry directions are
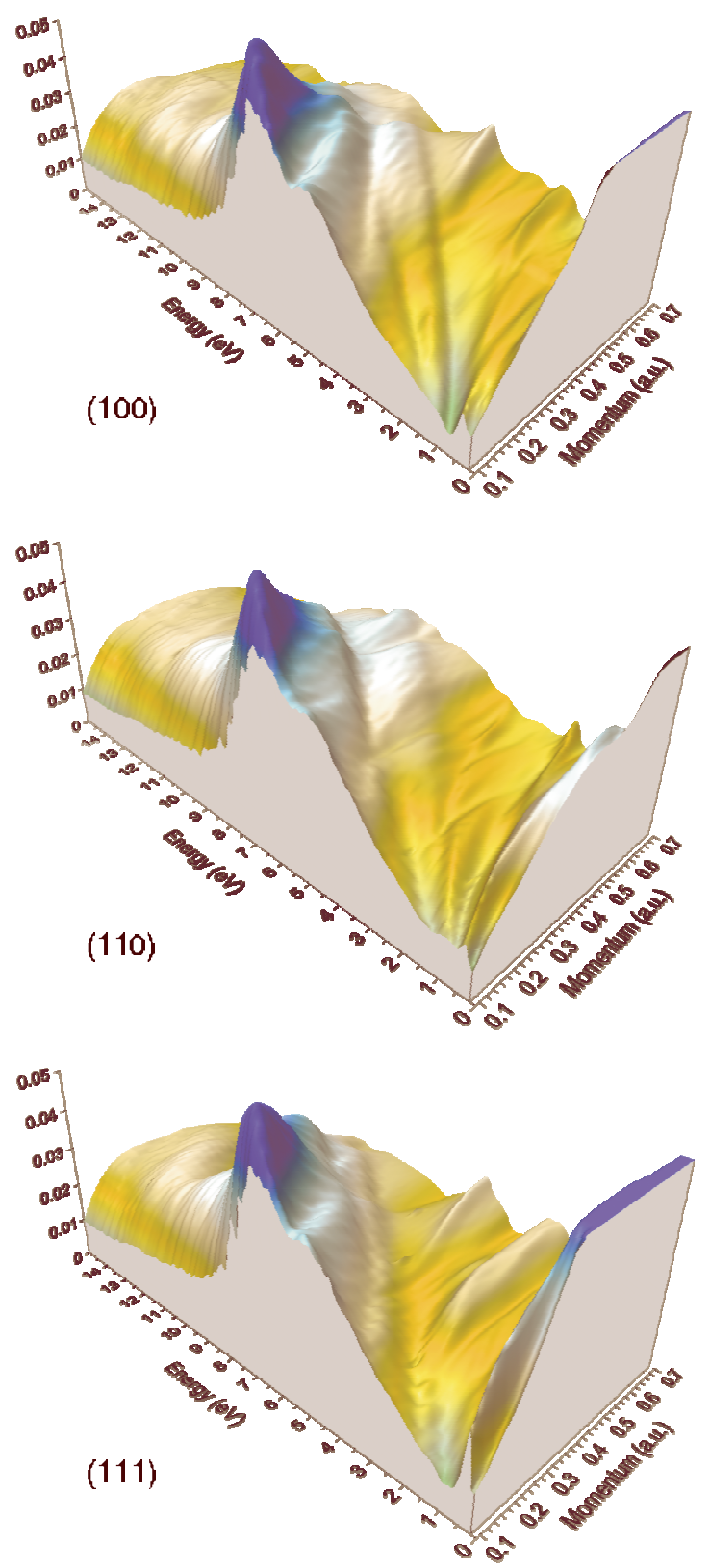

FIG. 2. (Color online) Normalized energy-loss spectrum $\operatorname{Im}\left[\epsilon_{\mathbf{G}=0, \mathbf{G}^{\prime}=0}^{-1}(\mathbf{q}, \omega)\right] / \omega\left(\right.$ in $\left.\mathrm{eV}^{-1}\right)$ for pure Pd as a function of $q$ and $\omega$ along the (100), (110), and (111) directions at top, middle, and bottom panels, respectively. Crystal local-field effects are included. The low-energy peaks were cut at $0.05 \mathrm{eV}^{-1}$.

dominated by the main broad feature centered at $\sim 7.2 \mathrm{eV}$ for the smallest momenta and at $\sim 8 \mathrm{eV}$ for $q \simeq 0.3$ a.u. $^{-1}$ The position of this peak at small $q$ is in good agreement with other $a b$ initio calculations ${ }^{16,39}$ and with the majority of values obtained in numerous optical experiments ${ }^{7,14}$ which, despite a rather large spread, place this peak around $7.3 \mathrm{eV}$. Our value of $\sim 8 \mathrm{eV}$ for larger wave vector is close to $8.2 \mathrm{eV}$ of Ref. 40 and $8.4 \mathrm{eV}$ of Ref. 15, where a significantly more scarce $\mathbf{k}$ point mesh was used in the $\chi^{0}$ evaluation. Also, we find a fairly good agreement with electron-energy-loss spectra measurements which place a dominating feature around $7 \mathrm{eV} \cdot{ }^{13}$ For small $q$, this peak is almost nondispersing in 


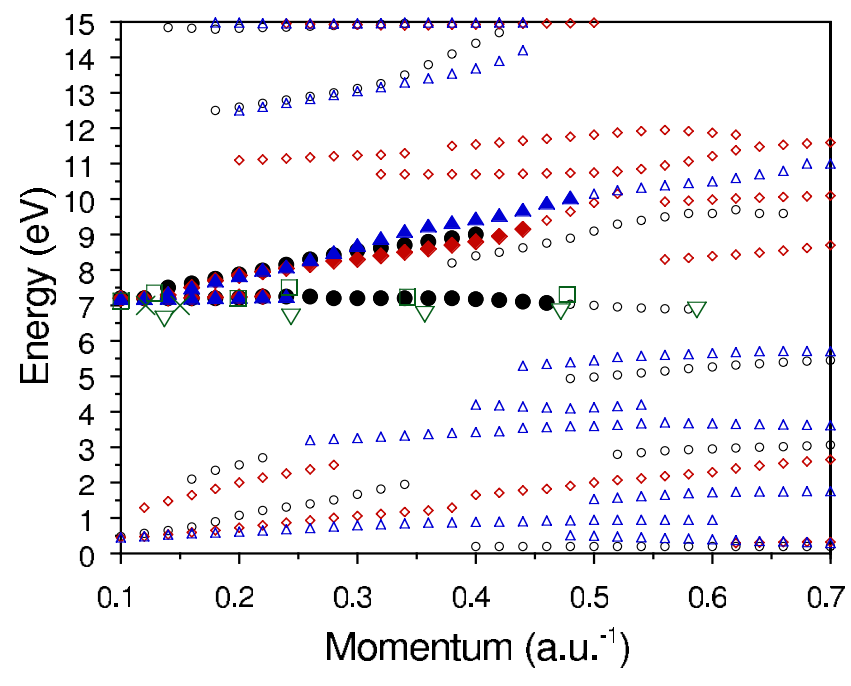

FIG. 3. (Color online) Peak structure positions in the calculated loss spectra of Pd shown in Fig. 2. The data along the (100), (110), and (111) symmetry directions are indicated by circles, diamonds, and triangles, respectively. The filled symbols refer to the dominant peak positions in the calculated loss spectra. The positions of dominant loss peaks in the experimental data are shown by crosses (Ref. 10), squares (Ref. 12), and inverted triangles (Ref. 18). Note that in the latter case, the peak positions are strongly affected by surface plasmon (Ref. 18).

agreement with electron-energy-loss investigations, ${ }^{10,12}$ but with increasing $q$, its absorption strength is gradually redistributed to the higher-energy peak. Thus, along the (100) direction, this nondispersing peak is dying at $q \simeq 0.45 \mathrm{a.u}^{-1}$, whereas another feature appears at $7.5 \mathrm{eV}$ for $q \simeq 0.15$ dispersing almost linearly with $q$ up to $\simeq 9 \mathrm{eV}$ at $q$ $\simeq 0.4$ a.u. ${ }^{-1}$ Along the (110) direction, the nondispersing part disappears at much smaller $q(\simeq 0.3$ a.u. $)$, and the dominating part consists of a linear-dispersing feature which is clearly visible up to $\omega \simeq 9 \mathrm{eV}$ at $q \simeq 0.45 \mathrm{a.u}^{-1}$ Along the (111) direction, similar to the (110) the nondispersing peak disappears at $q \simeq 0.25$ a.u. ${ }^{-1}$, and the dominating feature disperses linearly up to almost $10 \mathrm{eV}$ at $q \simeq 0.5 \mathrm{a} . \mathrm{u}^{-1}$. Whereas behavior of the lower nondispersing peak at $\simeq 7.2 \mathrm{eV}$ is in agreement with the energy-loss experiments, ${ }^{10-12}$ the upper dispersing part was apparently not revealed in the experiments.

The dominant feature at $\sim 7 \mathrm{eV}$ in loss spectra is normally attributed to a bulk plasmon, although, taking into account the number of valence electrons in palladium, $N_{v}=10$, the bulk plasmon energy calculated within a free-electron gas model should be significantly larger. A close inspection of Fig. 1 reveals that three bands cross the Fermi level, making possible a great number of interband transitions with energies close to the bulk plasmon energy. Therefore, the freeelectron gas model is clearly not adequate in the case of Pd. To address the role that intra- and interband transitions play in the formation of this plasmon peak, we have performed calculations taking into account only the intraband contributions in $\chi^{0}$. The calculation gives a Drude plasmon peak at $7 \mathrm{eV}$ for small $\mathbf{q}=\frac{2 \pi}{a_{\mathrm{Pd}}}\left(\frac{1}{48}, \frac{1}{48}, 0\right)$, which is in good agreement with the value of $7.1 \mathrm{eV}$ obtained in optical calculations. ${ }^{16,41}$

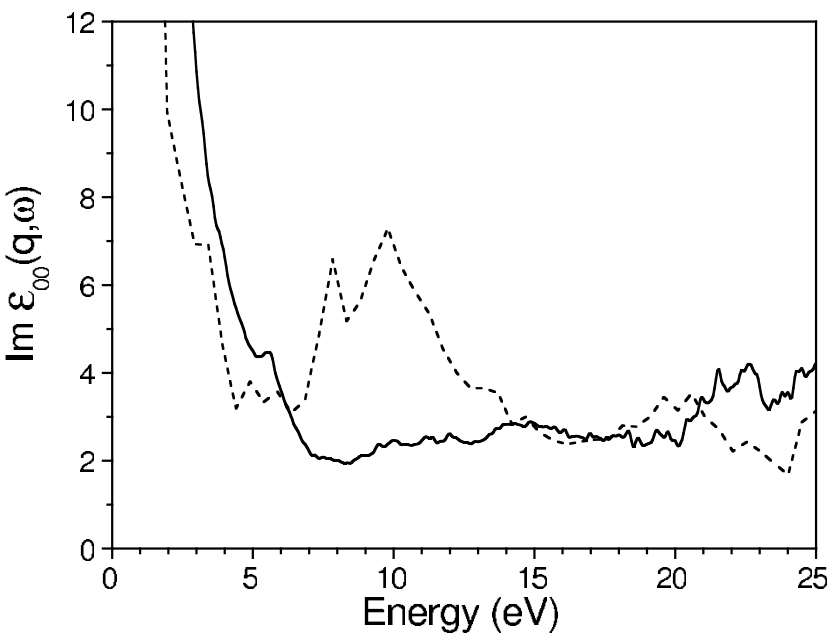

FIG. 4. The calculated imaginary part of dielectric function $\epsilon$ for $\mathrm{Pd}$ (solid line) for $\mathbf{q}=2 \pi / a_{\mathrm{Pd}}(1 / 8,0,0)$ and $\mathrm{PdH}$ (dashed line) for $\mathbf{q}=2 \pi / a_{\mathrm{PdH}}(1 / 8,0,0)$.

The excitation spectrum of Pd evaluated with the intraband transitions only is significantly modified by numerous interband transitions from the occupied $d$ bands to the unoccupied bands. The most efficient transitions from the band of $d$ symmetry should be to the states of $p$ and $f$ symmetries. The $p$ states are presented in the low-energy unoccupied part of the palladium electron-energy spectra, whereas the $f$ states are situated at significantly higher energies. This picture of transitions is corroborated by Fig. 4, where we place the imaginary part of $\epsilon_{\mathbf{G}=0, \mathbf{G}^{\prime}=0}(\mathbf{q}, \omega)$ for $\mathbf{q}=\frac{2 \pi}{a_{\mathrm{Pd}}}\left(\frac{1}{8}, 0,0\right)$ (solid line) which is very similar to those reported previously. ${ }^{16,42}$ This function reflects all one-particle transitions available in the electronic system and their strength. One can see that it does not contain sharp structures in the energy range from $\sim 7$ to $\sim 20 \mathrm{eV}$, whereas for higher energies, some peak features arise. Nevertheless, the enhanced values of $\operatorname{Im} \epsilon$ in this and upper energy regions do not change significantly the position around $\omega=7 \mathrm{eV}$ where the real part of $\epsilon$ crosses the $\omega=0$ line. ${ }^{16}$ This fact, together with the relatively low value of $\operatorname{Im} \epsilon$ at $\sim 7 \mathrm{eV}$, leads to the appearance of a pronounced peak in the loss function.

Additionally, we have decomposed the total $\operatorname{Im} \epsilon$ spectra into the partial contributions from the initial bands $i$ into the final bands $f$ in the same fashion as it was done by Lässer and Smith, ${ }^{42}$ and some of them are presented in Fig. 5. Comparing with the figures of Ref. 42, where detailed analysis of all transitions for these energies was performed, we observe rather good agreement with the data of Ref. 42. In Fig. 5, one can see that interband transitions from all the occupied bands (mainly of $d$ symmetry) to the unoccupied part of band 6 (mainly of $p$ symmetry) dominate contributions in the energy range from $\sim 2$ to $\sim 7 \mathrm{eV}$. The contributions from bands 3 , 4 , and 5 to band 7 (which has a large contribution of $p$ character) determine Im $\epsilon$ in the energy range from $\sim 7$ up to $\sim 13 \mathrm{eV}$. Hence, the peak in the loss function at $\sim 7 \mathrm{eV}$ is influenced by charge fluctuations between the occupied $d$ orbitals and the lowest unoccupied $p$ orbital.

In addition to the main peak, in Figs. 2 and 3, a clear peak is seen for large momenta and $\omega \rightarrow 0$ along all three symme- 


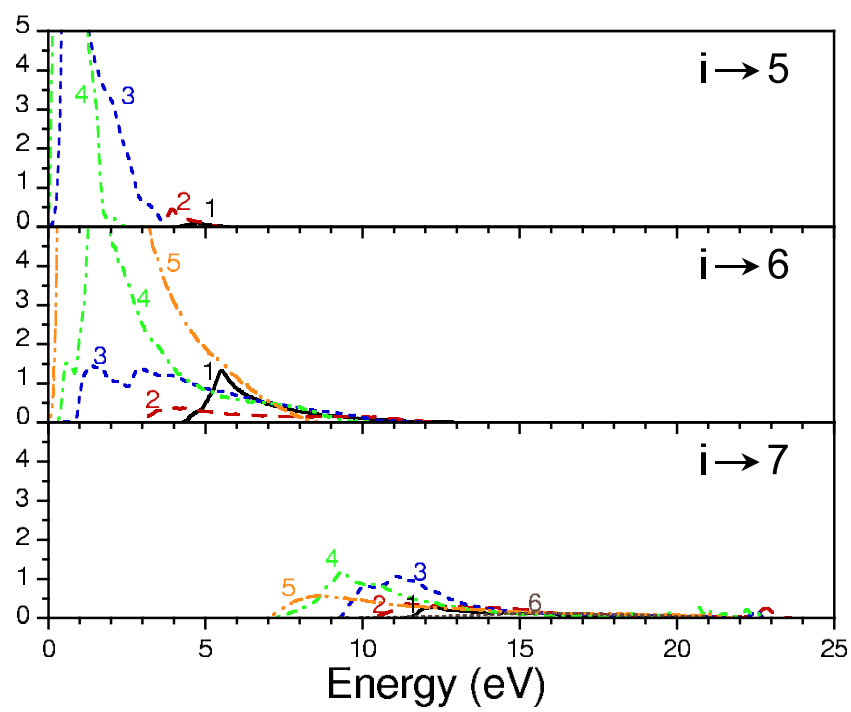

FIG. 5. (Color online) Partial contributions to $\operatorname{Im} \epsilon(\mathbf{q}, \omega)$, $\operatorname{Im} \epsilon^{i \rightarrow f}$ for transitions from the initial band $i$ into final band $f$ in the pure $\mathrm{Pd}$ case. The calculations are for $\mathbf{q}=2 \pi / a_{\mathrm{Pd}}(1 / 8,0,0)$. Each panel is labeled by the corresponding final state. In each panel, solid, long-dashed, dashed, dashed-dotted, dashed-double-dotted, and dotted lines correspond to transitions from zones 1, 2, 3, 4, 5, and 6 , respectively.

try directions. It occupies large phase space along the (111) direction being less pronounced in the (110) direction. Indeed, in all the directions for $\omega \rightarrow 0, \operatorname{Im} \epsilon^{-1}$ also goes to zero, but due to the chosen representation in Fig. 2, $\left(\operatorname{Im} \epsilon^{-1}\right) / \omega$ diverges, signaling that $\operatorname{Im} \epsilon^{-1}$ tends to zero slower than $\omega$. Some features corresponding to interband transitions can also be observed for large $q$ at $\simeq 4.3$ and $\simeq 5.5 \mathrm{eV}$ for the (100) and (111) directions, at $\simeq 2.5 \mathrm{eV}$ along the (110) direction, and below $2 \mathrm{eV}$ for all the directions. Also, in all the directions, some narrow peaks are present at $0.5 \mathrm{eV}$ for small momenta dispersing linearly with increasing $q$. A closer inspection of this feature shows that it is more pronounced along the (111) direction (where another small peak appears even for smaller energy) and is less visible along the (110) direction. In general, all these low-energy features reflect a complicated electronic structure of Pd around the Fermi level.

In order to investigate the role played by crystal localfield effects in Pd, we plot in Fig. 6 the loss function evaluated without inclusion of crystal local fields, i.e., $\operatorname{Im}\left[\epsilon_{\mathbf{G}=0, \mathbf{G}^{\prime}=0}(\mathbf{q}, \omega)\right]^{-1} / \omega$. Its comparison with Fig. 2 shows that the main effect of the inclusion of crystal local fields in $\mathrm{Pd}$ is the smoothing of sharp interband features for large $q$ without notable effect on the number and position of the peaks. This situation is similar to that observed in the $3 d$ metal series. ${ }^{36,43}$

\section{B. PdH}

Absorption of hydrogen causes significant modifications of the Pd band structure. ${ }^{8,44-48}$ In particular, hydrogen (1) lifts the Fermi level, (2) results in the formation of a mixed
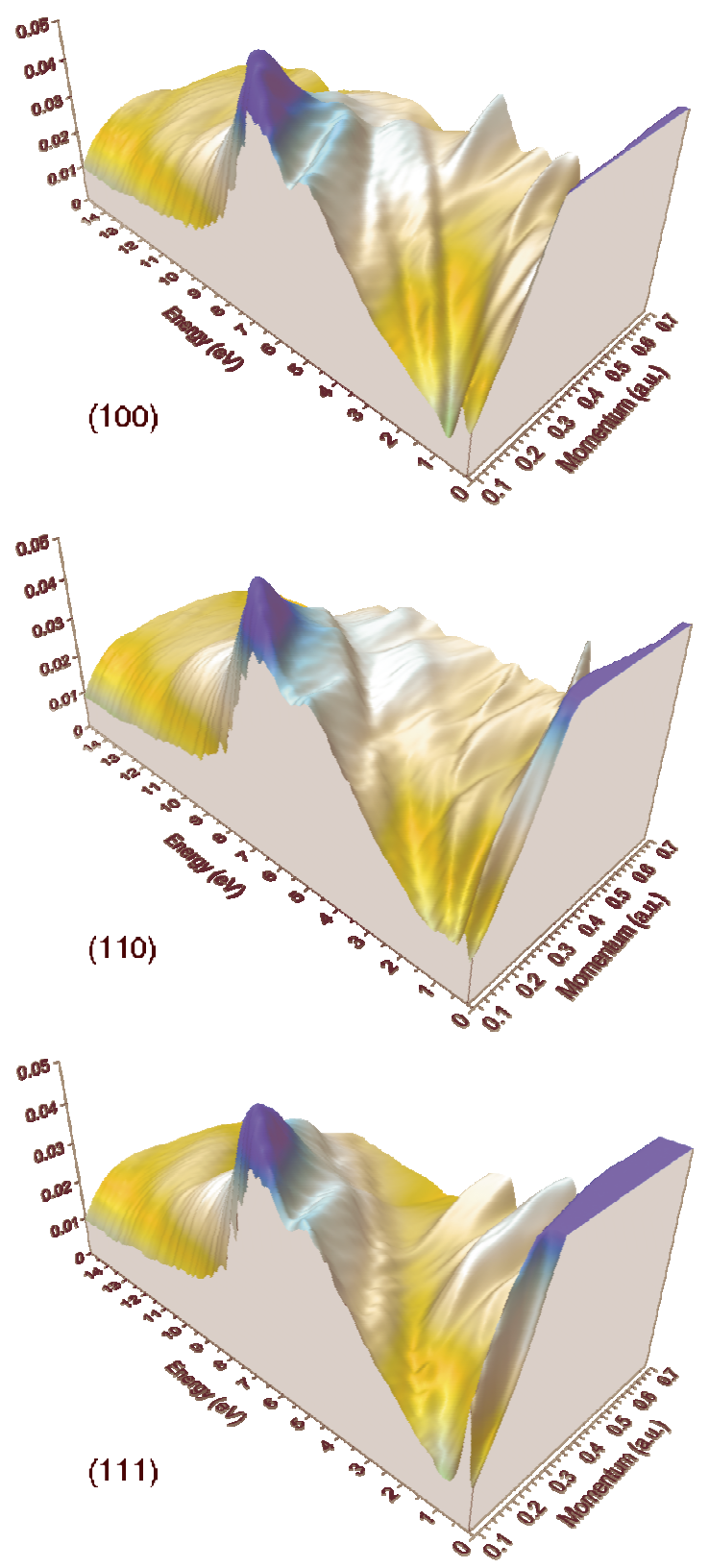

FIG. 6. (Color online) Normalized energy-loss spectrum $\operatorname{Im}\left[\epsilon_{\mathbf{G}=0, \mathbf{G}^{\prime}=0}(\mathbf{q}, \omega)\right]^{-1} / \omega\left(\right.$ in $\left.\mathrm{eV}^{-1}\right)$ for pure Pd as a function of $q$ and $\omega$ along the (100), (110), and (111) directions at top, middle, and bottom panels, respectively. Crystal local-field effects are not included. The low-energy peaks were cut at $0.05 \mathrm{eV}^{-1}$.

$\mathrm{Pd}-\mathrm{H}$ band at the bottom of valence band, and (3) gives rise to new bands above $E_{F}$. In Fig. 7, we show the evaluated band structure of PdH which is in good agreement with other calculations. ${ }^{8,48}$ As seen from the figure, all palladium $4 d$ bands in PdH are occupied and the Fermi level is crossed by the $s-p$ band only. This results in a much simpler Fermi surface in PdH compared to pure Pd. Another visible change of electronic structure is the position of the lowest occupied band which is about $2-3 \mathrm{eV}$ lower than the bottom of the pure Pd spectrum. This change is more pronounced at the $\Gamma$ point where the state has predominantly $s$ character and less pronounced at the BZ borders, where it is mainly of $d$ sym- 


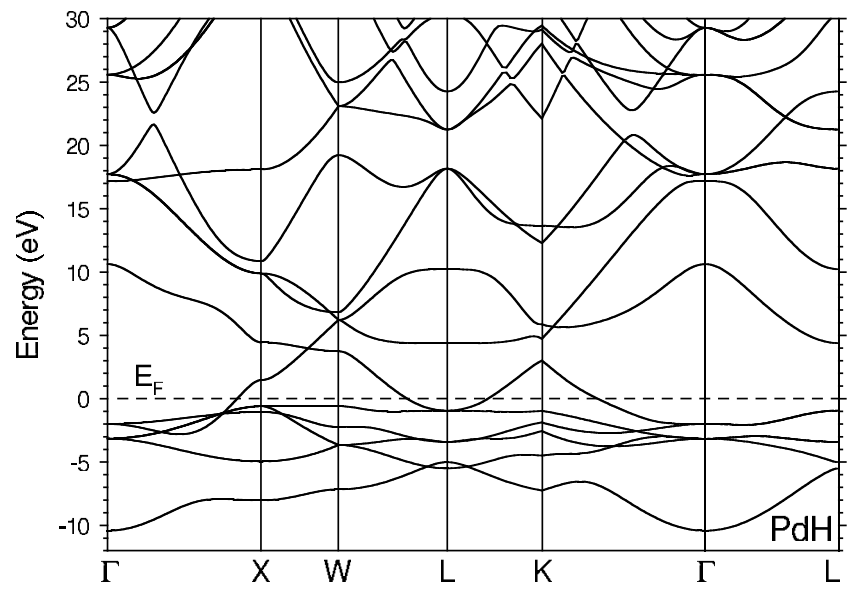

FIG. 7. The calculated band structure for PdH along some high symmetry directions. The energies are according to the Fermi level shown by dashed horizontal line.

metry. Also an unoccupied band appears at $\sim 10 \mathrm{eV}$ at the $\Gamma$ point which disperses downward toward the BZ borders. This leads to a significant reduction of a gap at the BZ borders between the top of $4 d$ band and band 6 which has predominantly $p$ symmetry. In pure $\mathrm{Pd}$, this band is situated at $\sim 24 \mathrm{eV}$ at the $\Gamma$ point. At the same time, comparison with Fig. 1 shows that the bands which have predominantly $d$ symmetry are affected by hydrogen at much less scale.

The $\mathrm{H}$ modifications of band structure of clean Pd lead to dramatic changes in the response function. This can be seen in Fig. 8 where the imaginary part of the inverse dielectric function for PdH is presented for the three symmetry directions of $\mathrm{BZ}$ [again, as in the pure $\mathrm{Pd}$ case, we plot $\operatorname{Im} \epsilon_{\mathbf{G}=0, \mathbf{G}^{\prime}=0}^{-1}(\mathbf{q}, \omega)$ scaled by $\omega^{-1}$ to emphasize to the low- $\omega$ domain]. The corresponding plot for the peak positions is shown in Fig. 9. The most prominent feature in the case of $\mathrm{PdH}$ is a dominant peak structure observed at $4.2 \mathrm{eV}$ for small momenta in all the directions. This peak is almost nondispersing and has a large amplitude up to $q \simeq 0.35$ a.u. $^{-1}$ from where it starts to gradually lose its strength. Nevertheless, along the (100) direction, it is clearly distinguished up to $q=0.7$ a.u. $^{-1}$. In Fig. 8, a well defined peak in $\left[\operatorname{Im} \epsilon^{-1}(\mathbf{q}, \omega)\right] / \omega$ arises at $\sim 3 \mathrm{eV}$ for small $q$, although the corresponding amplitude of $\operatorname{Im} \epsilon^{-1}(\mathbf{q}, \omega)$ is significantly lower than the amplitude of the main peak at $4.2 \mathrm{eV}$. This feature disperses upward almost linearly with $q$ in all three directions and disappears at $q \simeq 0.2$ a.u. $^{-1}$ Even for lower energies, a weak peak is visible in the (100) direction. For small $q$, it is located at $\simeq 1 \mathrm{eV}$ and disperses upward linearly with increasing $q$. The downward shift of the main peak structure to 3-6 eV region correlates very well with the experimental loss peak at $\sim 5 \mathrm{eV} .{ }^{5}$ As all the experiments have been performed for substoichiometric $\mathrm{PdH}_{x}$ with $x<1$, it would be desirable to perform EELS measurements for stoichiometric PdH samples for direct comparisons of ab initio and experimental results.

A low density of states at the Fermi level in PdH leads to dramatic reduction of the intensity of $\operatorname{Im} \epsilon^{-1}$ for small energies. Thus, instead of a large value of $\left[\operatorname{Im} \epsilon^{-1}(\mathbf{q}, \omega)\right] / \omega$ for $\omega \rightarrow 0$, a significant depression is visible in $\mathrm{PdH}$ for all mo-
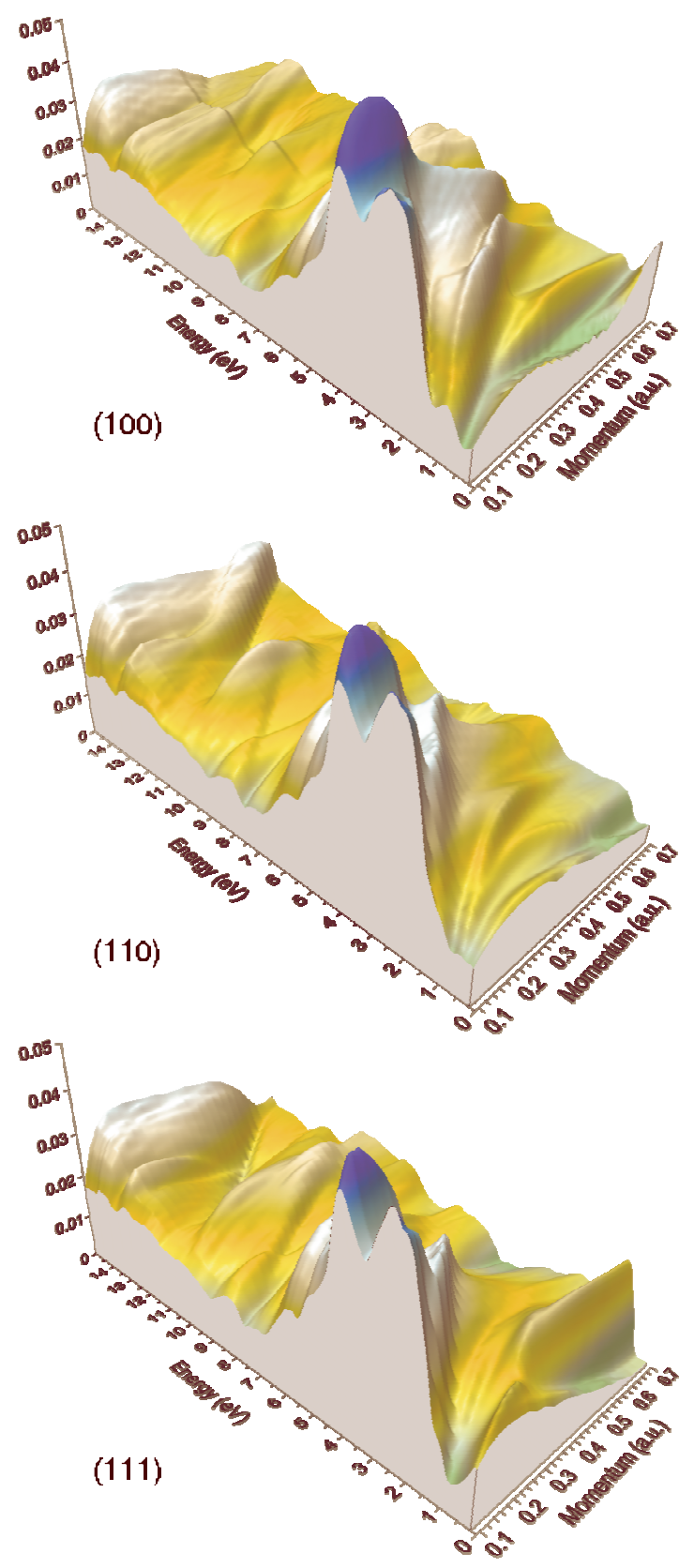

FIG. 8. (Color online) Normalized energy-loss spectrum $\operatorname{Im}\left[\epsilon_{\mathbf{G}=0, \mathbf{G}^{\prime}=0}^{-1}(\mathbf{q}, \omega)\right] / \omega\left(\right.$ in $\left.\mathrm{eV}^{-1}\right)$ for $\mathrm{PdH}$ as a function of $q$ and $\omega$ along the (100), (110), and (111) directions at top, middle, and bottom panels, respectively. Crystal local-field effects are included.

menta and the threshold for interband transitions from occupied $d$ bands at large momenta is observed at energies of 0.8 , 0.6 , and $0.5 \mathrm{eV}$ for the (100), (110), and (111) directions, respectively. Additionally, two less pronounced nondispersing peaks appear at $\omega \simeq 5 \mathrm{eV}$ for $q \leqslant 0.3$ a.u. $^{-1}$ and $\omega$ $\simeq 6 \mathrm{eV}$ for $q \leqslant 0.5 \mathrm{a}^{-u}{ }^{-1}$. Along the (100) direction, a slightly dispersing upward feature appears at $\omega \simeq 7 \mathrm{eV}$ for $q \geqslant 0.4$ a.u. $^{-1}$. In the higher-energy region, a noticeable peak arises at $\simeq 8.2 \mathrm{eV}$ for $q<0.25$ a.u. $^{-1}$ in all the directions. A strong broad almost flat feature is located at $\omega \simeq 10.5 \mathrm{eV}$. It is more pronounced along the (110) and (111) directions and has the maximum intensity for 0.2 a.u. ${ }^{-1} \leqslant q \leqslant 0.5$ a.u. ${ }^{-1}$. 


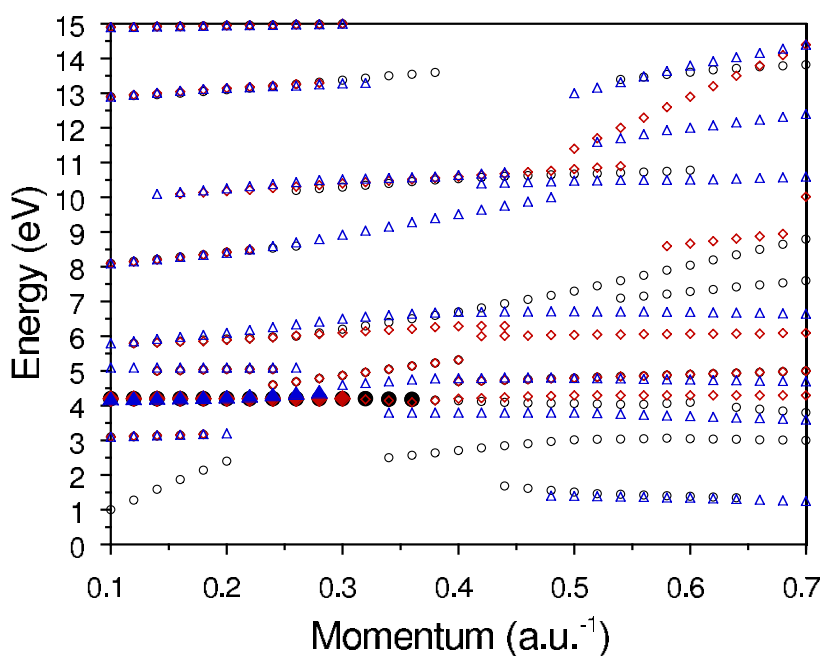

FIG. 9. (Color online) Peak structure positions in the calculated loss spectra of PdH shown in Fig. 8. The data along the (100), (110), and (111) symmetry directions are indicated by circles, diamonds, and triangles, respectively. The filled symbols refer to the dominant peak positions in the calculated loss spectra.

The strong structure appears for energies higher than $12 \mathrm{eV}$.

We calculated the bare plasmon energy (Drude peak) in $\mathrm{PdH}$ taking into account in the evaluation of $\chi^{0}$ the intraband contributions corresponding to band 6 only. The Drude plasmon energy of $6.8 \mathrm{eV}$ calculated for $\mathbf{q}=\frac{2 \pi}{a_{\mathrm{PdH}}}\left(\frac{1}{24}, \frac{1}{24}, 0\right)$ is only

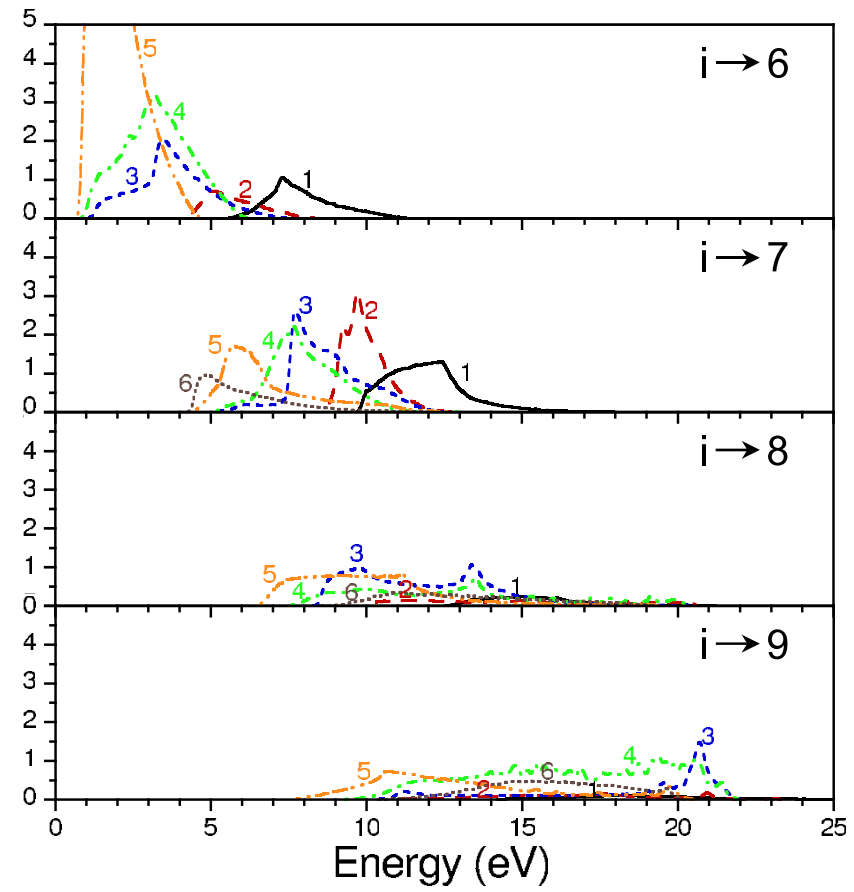

FIG. 10. (Color online) Partial contributions to $\operatorname{Im} \epsilon(\mathbf{q}, \omega)$, $\operatorname{Im} \epsilon^{i \rightarrow f}$ for transitions from the initial band $i$ into final band $f$ in the $\mathrm{PdH}$ case. The calculations are for $\mathbf{q}=2 \pi / a_{\mathrm{PdH}}(1 / 8,0,0)$. Each panel is labeled by the corresponding final state. In each panel, solid, long-dashed, dashed, dashed-dotted, dashed-double-dotted, and dotted lines correspond to transitions from zones 1, 2, 3, 4, 5, and 6 , respectively.
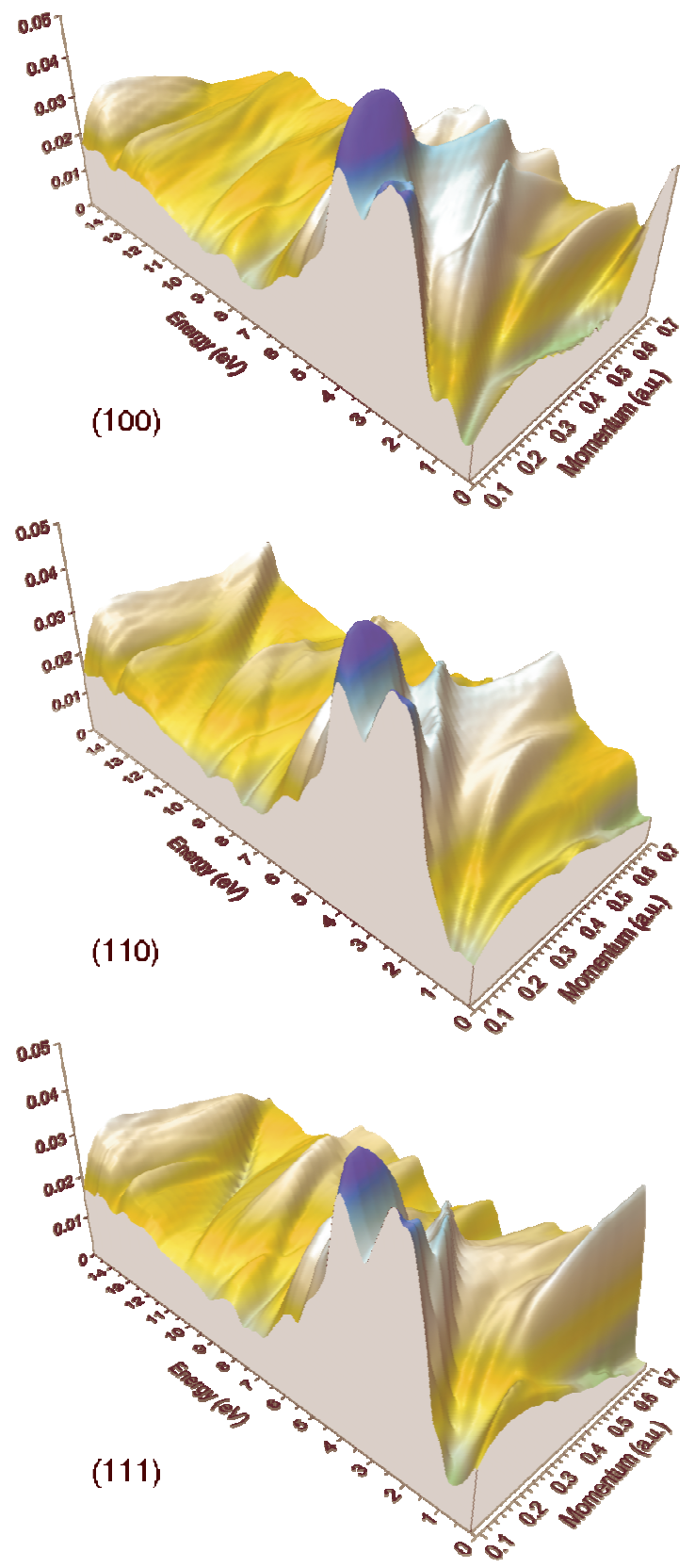

FIG. 11. (Color online) Normalized energy-loss spectrum $\operatorname{Im}\left[\epsilon_{\mathbf{G}=0, \mathbf{G}^{\prime}=0}(\mathbf{q}, \omega)\right]^{-1} / \omega\left(\right.$ in $\left.\mathrm{eV}^{-1}\right)$ for PdH as a function of $q$ and $\omega$ along the (100), (110), and (111) directions at top, middle, and bottom panels, respectively. Crystal local-field effects are not included.

$0.2 \mathrm{eV}$ smaller than the value obtained for Pd. Hence, it cannot explain the observed shift of plasmon peak from $7.2 \mathrm{eV}$ in $\mathrm{Pd}$ to $4.2 \mathrm{eV}$ in $\mathrm{PdH}$. In order to investigate the origin of this significant downward shift of the low-energy dominant peak structure in $\mathrm{PdH}$ in comparison with pure $\mathrm{Pd}$, we plot in Fig. 4 the $\operatorname{Im} \epsilon(\mathbf{q}, \omega)$ for $\mathbf{q}=\frac{2 \pi}{a_{\mathrm{PdH}}}\left(\frac{1}{8}, 0,0\right)$. One can see that in $\mathrm{PdH}$ in contrast to pure $\mathrm{Pd}$, an additional sharp two-peak structure in $\operatorname{Im} \epsilon$ arises in the energy range from 7 to $14 \mathrm{eV}$. Due to interrelation between imaginary and real parts of the dielectric function via the Hilbert transform, this additional feature pushes the real part of the dielectric function to cross 
the $\omega=0$ axis at the much lower energy. In Fig. 10, we plot the contributions to $\operatorname{Im} \epsilon$ in $\mathrm{PdH}$ which are relevant for this energy range. Comparing Figs. 10 and 5, one can see the shift to higher energies of the low edge of all transitions to band 6 . One can also see that the contribution due to the 1 $\rightarrow 6$ transitions maintains, in general, its shape and strength, while the contributions from bands 4 and 5 are significantly reduced. These effects produce, in $\mathrm{PdH}$, the drastic decrease of $\operatorname{Im} \epsilon$ for energies below $6 \mathrm{eV}$ with respect to that in $\mathrm{Pd}$, Fig. 4. The contributions from the $i \rightarrow 7$ transitions are significantly stronger in $\mathrm{PdH}$ than in $\mathrm{Pd}$, and they are shifted to lower energies by $2-3 \mathrm{eV}$. These contributions strongly enhance $\operatorname{Im} \epsilon$ at $7-14 \mathrm{eV}$, although some minor contributions result from the $i \rightarrow 8$ and $i \rightarrow 9$ transitions. The $1 \rightarrow 7$ transitions contribute for $\omega>10 \mathrm{eV}$ and therefore have less impact on $\operatorname{Re} \epsilon$ in comparison with the other $i \rightarrow 7$ transitions. Therefore, one can account for the shift of the main peak in the PdH loss function to $4.2 \mathrm{eV}$ as a result of fluctuating density oscillations between the occupied $\mathrm{Pd} 4 d$ bands (only slightly modified by $\mathrm{H}^{8}$ ) and the strongly modified unoccupied $p$ band, although transitions from the lowest mixed Pd-H band to the unoccupied part of band 6 (of $p$ character) have some impact.

Figure 11 shows loss function for $\mathrm{PdH}$ without the inclusion of crystal local-field effects. The comparison of Fig. 11 with Fig. 8 reveals that, as in the case of pure Pd, the inclusion of these effects results in some changes of the amplitude of Im $\epsilon^{-1}$ especially for large $q$ but does not produce a significant effect on the peak positions. Nevertheless, the comparison of Figs. 2 and 6 and of Figs. 8 and 11 reveal that the crystal local fields modify the loss function shape in $\mathrm{PdH}$ stronger than in Pd.

\section{CONCLUSIONS}

We have presented a theoretical study of electronic excitation spectra in Pd and PdH based on ab initio calculations of band structure of these materials. It has been shown that complicated band structures in both systems are responsible for the rich structure observed in the excitation spectra. Good agreement with available experimental optical and energyloss experiments and other theoretical studies is obtained for small momenta in Pd. The various peak features are obtained in Pd for momenta up to $q=0.7$ a.u. ${ }^{-1}$. All the calculated features show slight momentum anisotropy.

We have demonstrated that energy-loss spectra in $\mathrm{PdH}$ is strongly modified with respect to that in $\mathrm{Pd}$ for the entire $q$ region studied. The main plasmon peak in the PdH loss function is situated at $\simeq 4.2 \mathrm{eV}$ and has almost flat dispersion in all three symmetry directions up to $q=0.7$ a.u. ${ }^{-1}$ with gradual reduction of amplitude for $q>0.4$ a.u. ${ }^{-1}$. We have studied the origin of the pronounced shift of the main plasmon peak from $\simeq 7.2 \mathrm{eV}$ in $\mathrm{Pd}$ to $\simeq 4.2 \mathrm{eV}$ in $\mathrm{PdH}$ and found that it is mainly due to interband transitions in $\mathrm{PdH}$ from occupied $\operatorname{Pd} d$ bands to hydrogen-modified unoccupied $p$ band, although some effect is due to transitions from the lowest occupied Pd-H band to the lowest $p$ unoccupied band.

In both systems studied, the crystal local-field effects are shown to play only a minor role in the formation of the loss function.

\section{ACKNOWLEDGMENTS}

We acknowledge the partial support from the University of the Basque Country (9/UPV 00206.215-13639/2001), the Departamento de Educación del Gobierno Vasco, and the Spanish Ministerio de Ciencia y Tecnología (MCyT) (FIS 2004-06490-C03-01).
${ }^{1}$ W. M. Muller, J. P. Blackledge, and G. G. Libowitz, Metal Hydrides (Academic, New York, 1968).

${ }^{2}$ Hydrogen in Metals I, edited by G. Alefeld and J. Völk1, Topics in Applied Physics, Vol. 28 (Springer, Berlin, 1978); Hydrogen in Metals II, edited by G. Alefeld and J. Völkl, Topics in Applied Physics, Vol. 29 (Springer, Berlin, 1978).

${ }^{3}$ P. V. Geld, P. A. Ryabov, and L. P. Mohracheva, Hydrogen and Physical Properties of Metals and Alloys (Nauka, Moscow, 1985).

${ }^{4}$ F. A. Lewis, The Palladium Hydrogen System (Academic, New York, 1967).

${ }^{5}$ P. A. Bennett and J. C. Fuggle, Phys. Rev. B 26, 6030 (1982).

${ }^{6}$ S. Mizusaki, T. Miyatake, N. Sato, I. Yamamoto, M. Yamaguchi, M. Itou, and Y. Sakurai, Phys. Rev. B 73, 113101 (2006).

${ }^{7}$ J. H. Weaver, Phys. Rev. B 11, 1416 (1975).

${ }^{8}$ C. T. Chan and S. G. Louie, Phys. Rev. B 27, 3325 (1983).

${ }^{9}$ N. E. Christensen, Phys. Rev. B 14, 3446 (1976).

${ }^{10}$ G. Chiarello, E. Colavita, M. De Crescenzi, and S. Nannarone, Phys. Rev. B 29, 4878 (1984).

${ }^{11}$ M. Nishijima, M. Jo, Y. Kuwahara, and M. Onchi, Solid State Commun. 58, 75 (1986)

${ }^{12}$ T. Bornemann, J. Eickmans, and A. Otto, Solid State Commun.
65, 381 (1988).

${ }^{13}$ H. A. E. Hagelin, J. F. Weaver, G. B. Hoflund, and G. N. Salaita, J. Electron Spectrosc. Relat. Phenom. 124, 1 (2002), and references therein.

${ }^{14}$ Handbook of Optical Constants of Solids, edited by E. D. Palik (Academic, San Diego, 1991).

${ }^{15}$ E. G. Maksimov, I. I. Mazin, S. N. Rashkeev, and Yu. A. Uspenski, J. Phys. F: Met. Phys. 18, 833 (1988).

${ }^{16}$ E. E. Krasovskii and W. Schattke, Phys. Rev. B 63, 235112 (2001).

${ }^{17}$ V. P. Zhukov, F. Aryasetiawan, E. V. Chulkov, and P. M. Echenique, Phys. Rev. B 65, 115116 (2002).

${ }^{18}$ F. P. Netzer and M. M. El Gomati, Surf. Sci. 124, 26 (1983).

${ }^{19}$ A. C. Switendick, Ber. Bunsenges. Phys. Chem. 76, 535 (1972).

${ }^{20}$ D. A. Papaconstantopoulos, B. M. Klein, J. S. Faulkner, and L. L. Boyer, Phys. Rev. B 18, 2784 (1978).

${ }^{21}$ A. Harmalkar, D. G. Kanhere, and R. M. Singru, Phys. Rev. B 31, 6415 (1985).

${ }^{22}$ M. Gupta and A. J. Freeman, Phys. Rev. B 17, 3029 (1978).

${ }^{23}$ M. J. van Setten, V. A. Popa, G. A. de Wijs, and G. Brocks, Phys. Rev. B 75, 035204 (2007).

${ }^{24}$ C. Moysés Araújo, S. Lebègue, O. Eriksson, B. Arnaud, M. Al- 
ouani, and R. Ahuja, J. Appl. Phys. 98, 096106 (2005).

${ }^{25}$ O. V. Slobodyan and E. E. Krasovskii, Int. J. Hydrogen Energy 20, 361 (1995).

${ }^{26}$ D. Pines and P. Nozières, The Theory of Quantum Liquids (Benjamin, New York, 1966), Vol. 1.

${ }^{27}$ E. Runge and E. K. U. Gross, Phys. Rev. Lett. 52, 997 (1984).

${ }^{28}$ M. Petersilka, U. J. Gossmann, and E. K. U. Gross, Phys. Rev. Lett. 76, 1212 (1996).

${ }^{29}$ W. Ku and A. G. Eguiluz, Phys. Rev. Lett. 82, 2350 (1999).

${ }^{30}$ N. E. Maddocks, R. W. Godby, and R. J. Needs, Europhys. Lett. 27, 681 (1994).

${ }^{31}$ F. Aryasetiawan and O. Gunnarsson, Phys. Rev. B 49, 16214 (1994).

${ }^{32}$ F. Aryasetiawan, in Strong Coulomb Correlations in Electronic Structure Calculations, edited by V. I. Anisimov (Gordon and Beach, Singapore, 2001).

${ }^{33}$ D. K. Ross, V. E. Antonov, E. L. Bokhenkov, A. I. Kolesnikov, E. G. Ponyatovsky, and J. Tomkinson, Phys. Rev. B 58, 2591 (1998).

${ }^{34}$ D. M. Ceperley and B. J. Alder, Phys. Rev. Lett. 45, 566 (1980), as parametrized by J. P. Perdew and A. Zunger, Phys. Rev. B 23, 5048 (1981).
${ }^{35}$ N. Troullier and J. L. Martins, Phys. Rev. B 43, 1993 (1991).

${ }^{36}$ I. G. Gurtubay, J. M. Pitarke, W. Ku, A. G. Eguiluz, B. C. Larson, J. Tischler, P. Zschack, and K. D. Finkelstein, Phys. Rev. B 72, 125117 (2005).

${ }^{37}$ H. Chen, N. E. Brener, and J. Callaway, Phys. Rev. B 40, 1443 (1989).

${ }^{38}$ N. V. Smith, Phys. Rev. B 9, 1365 (1974).

${ }^{39}$ V. J. Keast, J. Electron Spectrosc. Relat. Phenom. 143, 97 (2005).

${ }^{40}$ G.-M. Fehrenbach, Phys. Rev. B 59, 15085 (1999).

${ }^{41}$ E. E. Krasovskii (private communication).

${ }^{42}$ R. Lässer and N. V. Smith, Phys. Rev. B 25, 806 (1982).

${ }^{43}$ I. G. Gurtubay, W. Ku, J. M. Pitarke, and A. G. Eguiluz, Comput. Mater. Sci. 30, 104 (2004).

${ }^{44}$ A. C. Switendick, Solid State Commun. 8, 1463 (1970).

${ }^{45}$ D. E. Eastman, J. K. Cashion, and A. C. Switendick, Phys. Rev. Lett. 27, 35 (1971).

${ }^{46}$ C. D. Gelatt, Jr., H. Ehrenreich, and J. A. Weiss, Phys. Rev. B 17, 1940 (1978).

${ }^{47}$ J. S. Faulkner, Phys. Rev. B 13, 2391 (1976).

${ }^{48}$ D. A. Papaconstantopoulos, B. M. Klein, E. N. Economou, and L. L. Boyer, Phys. Rev. B 17, 141 (1978). 